\title{
EM-BASED RECEIVER DESIGN OF LDPC-CODED MIMO SYSTEMS OVER UNKNOWN FADING CHANNELS
}

\author{
Jun Zheng and Bhaskar D. Rao \\ University of California at San Diego \\ juzheng@ucsd.edu,brao@ece.ucsd.edu
}

\begin{abstract}
We consider an LDPC-coded MIMO system operating in a fading environment, where channel state information is assumed to be unavailable both to the transmitter and the receiver. We propose at the component level a modified EM-based MIMO detector, which completely removes positive feedback between input and output extrinsic information and provide much better performance as compared to the regular EM-based detector that has strong correlations. At the structural level, motivated by the turbo iterative decoding strategy, a new LDPC-coded MIMO receiver is constructed in a novel manner where the soft MIMO detector and LDPC variable node decoder form one super soft decoding unit and the LDPC check node decoder the other component of the iterative decoding scheme. Based on the analysis of the extrinsic information transfer characteristic of the component soft decoders, a simple and efficient LDPC code optimization approach is also provided. Numerical and simulation results are provided to further confirm the optimality of the proposed design approach for the coded MIMO system.
\end{abstract}

\section{INTRODUCTION}

Communication systems using multiple antennas at both the transmitter and the receiver has recently received increased attention due to its promise of providing significant capacity increases in a wireless fading environment, as reported by Telatar [1]. However, various capacity analysis and MIMO system design provided so far is based on the underlying assumption that the fading channel coefficients between each transmit and receive antenna pairs are perfectly known at the receiver without any cost, which is not a reasonable assumption for most practical communication systems especially in fast fading channels.

For communication systems with unknown channel state information (CSIR) at the receiver, typically receivers have a two-phase structure, channel estimation using the preset training symbols followed by coherent data detection. However, conventional channel estimators form estimation only based on the training symbols, thereby not making use of

This research was supported by CoRe grant No. 02-10109 sponsored by Ericsson. the channel information contained in the received data symbols. Therefore, the two-phase model itself is limiting the performance and can not approach the MIMO channel capacity (or the maximum achievable information rate). On the other hand, the EM-based joint channel estimation and data detection algorithm [2][3][4], which can iteratively improve its channel estimation based on the soft extrinsic information from the outer soft decoders is promising and has recently received much attention.

The main contributions of this paper fall into two categories. At the component level, we propose in this paper a modified EM-based MIMO detector, which completely removes positive feedback between input and output extrinsic information and provide much better performance as compared to the regular EM-based detector that has strong correlations. Further at the structural level, a new coded MIMO receiver is constructed in a novel manner where the soft MIMO detector and LDPC variable node decoder form one super soft decoding unit and the LDPC check node decoder the other component of the iterative decoding scheme. Exploiting the new receiver structure, we also provide in this article a simple and efficient algorithm for the LDPC code degree profile optimization. Numerical and simulation results of the unknown LDPC-coded MIMO system using the optimized degree profile further confirm the optimality of the proposed design approach for the coded MIMO system.

\section{SYSTEM MODEL}

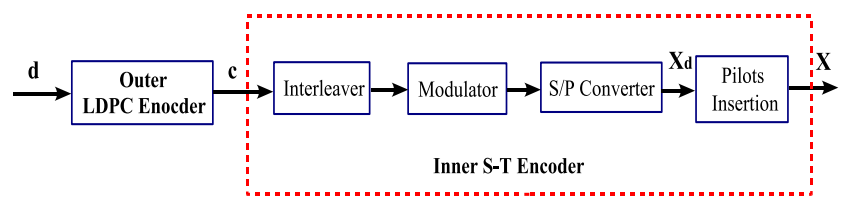

Fig. 1. Transmitter model of LDPC-coded MIMO systems

We consider in this paper a MIMO system with $M$ transmit antennas and $N$ receive antennas, signaling through a frequency flat fading channel with i.i.d channel propagation coefficient between each transmit and receive antenna pairs. As illustrated in Fig. 1, a block of $k$ binary information bits denoted $\mathbf{d}=\left\{d_{1}, \cdots, d_{k}\right\}$ is first encoded by 
an outer LDPC encoder with code rate $R=k / n$ into a codeword $\mathbf{c}=\left\{c_{1}, \cdots, c_{n}\right\}$ of length $n$. The codeword $\mathbf{c}$ is further segmented into $L$ consecutive sub-blocks $\mathbf{C}_{i}$ of length $K$. Each sub-block $\mathbf{C}_{i}$ is then encoded by the inner space-time encoder, which is composed of interleaver, modulator, serial-to-parallel converter, and pilots insertion operator, into a coherent sub-frame $\mathbf{X}_{i}$. The transmitted signal $\mathbf{X}_{i}$ can be stacked and partitioned into two sub-matrixes: training followed by data, which is represented as

$$
\mathbf{X}_{i}=\sqrt{\frac{\rho}{M}} \cdot\left[\mathbf{S}_{\tau}^{H}, \mathbf{X}_{d}^{i H}\right]^{H}
$$

where $\rho$ is the average signal to noise ratio at each receive antenna, $\mathbf{S}_{\tau}$ is the fixed pilot symbols and $\mathbf{X}_{d}^{i}$ is the information bearing data symbols, whose structures are given by

$$
\begin{array}{cl}
\mathbf{S}_{\tau}=\left[\mathbf{s}_{1}^{H}, \cdots, \mathbf{s}_{T_{\tau}}^{H}\right]^{H}, & \mathbf{S}_{\tau} \in \mathbb{C}^{T_{\tau} \times M}, \\
\mathbf{X}_{d}^{i}=\left[\mathbf{x}_{1}^{i}, \cdots, \mathbf{x}_{T_{d}}^{i}{ }^{H}\right]^{H}, & \mathbf{X}_{d}^{i} \in \mathbb{C}^{T_{d} \times M},
\end{array}
$$

with the following constraints

$$
\operatorname{tr}\left(\mathbf{S}_{\tau}^{H} \cdot \mathbf{S}_{\tau}\right)=M T_{\tau}, \quad E_{\mathbf{X}_{d}^{i}}\left[\operatorname{tr}\left(\mathbf{X}_{d}^{i}{ }^{H} \cdot \mathbf{X}_{d}^{i}\right)\right]=M T_{d} .
$$

Each element of the transmitted data signal $\mathbf{X}_{d}^{i}$ comes from a finite complex alphabet $\mathcal{X}$ of size $|\mathcal{X}|$.

It is assumed that the fading coefficient $\mathbf{H}_{i}$ remains static within each coherent sub-blocks and varies independently from one sub-block to another; and each element of $\mathbf{H}_{i}$ is complex Gaussian distributed. Hence, the signal model can be written as

$$
\mathbf{Y}_{i}=\mathbf{X}_{i} \cdot \mathbf{H}_{i}+\mathbf{w}_{i}, \quad 1 \leq i \leq L,
$$

where $\mathbf{Y}_{i}$ is a $T \times N$ received complex signal matrix, $\mathbf{X}_{i}$ is a $T \times M$ transmitted complex signal matrix, $\mathbf{H}_{i}$ is a $M \times$ $N$ complex channel matrix, and $\mathbf{w}_{i}$ is a $T \times N$ matrix of additive noise. Both matrix $\mathbf{H}_{i}$ and $\mathbf{w}_{i}$ have zero mean unit variance independent complex Gaussian entries.

\section{MODIFIED EM-BASED SOFT MIMO DETECTOR}

Instead of forming channel estimation based only on the training symbols, we apply a MAP EM estimator to iteratively improve its estimate based on the input a priori information from the outer soft decoder. By viewing the transmitted data signal $\mathbf{X}_{d}^{i}$ (or $\mathbf{X}_{i}$ ) as the unobserved (or missing) data, the maximum a posteriori probability (MAP) channel estimation $\widehat{\mathbf{H}}_{i}$ is obtained by the following EM algorithm,

\section{- E-step:}

$$
\begin{array}{r}
Q\left(\mathbf{H}_{i} \mid \widehat{\mathbf{H}}_{i}^{(k)}\right)=E_{\mathbf{X}_{i} \mid \widehat{\mathbf{H}}_{i}^{(k)}, \mathbf{Y}_{i}}\left[-\log p\left(\mathbf{H}_{i}, \mathbf{Y}_{i} \mid \mathbf{X}_{i}\right)\right] \\
=\operatorname{tr}\left(\mathbf{H}_{i}^{H} \mathbf{R} \mathbf{H}_{i}+\mathbf{Y}_{i}^{H} \mathbf{Y}_{i}-2 \Re\left[\mathbf{Y}_{i}^{H} \mathbf{U} \mathbf{H}_{i}\right]\right),
\end{array}
$$

where $\mathbf{R}$ and $\mathbf{U}$ are given by

$$
\begin{aligned}
\mathbf{R} & =\frac{\rho}{M}\left(\sum_{j=1}^{T_{d}} \sum_{\mathbf{x}_{j}^{i}} p\left(\mathbf{x}_{j}^{i} \mid \widehat{\mathbf{H}}_{i}^{(k)}, \mathbf{y}_{i}\right) \cdot \mathbf{x}_{j}^{i}{ }^{H} \mathbf{x}_{j}^{i}+\mathbf{S}_{\tau}^{H} \mathbf{S}_{\tau}\right)+I_{M}, \\
\mathbf{U} & =\sqrt{\frac{\rho}{M}}\left[\mathbf{S}_{\tau}^{H}, \mu_{1}^{H}, \cdots, \mu_{T_{d}}^{H}\right]^{H}, \mu_{j}=E\left[\mathbf{x}_{j}^{i} \mid \widehat{\mathbf{H}}_{i}^{(k)}, \mathbf{y}_{i}\right] .
\end{aligned}
$$

And the a posterior probability $p\left(\mathbf{x}_{j}^{i} \mid \widehat{\mathbf{H}}_{i}^{(k)}, \mathbf{y}_{i}\right)$ is given by

$$
p\left(\mathbf{x}_{j}^{i} \mid \widehat{\mathbf{H}}_{i}^{(k)}, \mathbf{y}_{i}\right)=\frac{p\left(\mathbf{y}_{i} \mid \widehat{\mathbf{H}}_{i}^{(k)}, \mathbf{x}_{j}^{i}\right) \cdot p\left(\mathbf{x}_{j}^{i}\right)}{\sum_{\mathbf{x}_{j}^{i} \in \mathcal{X}^{M}} p\left(\mathbf{y}_{i} \mid \widehat{\mathbf{H}}_{i}^{(k)}, \mathbf{x}_{j}^{i}\right) \cdot p\left(\mathbf{x}_{j}^{i}\right)}
$$

with $p\left(\mathbf{y}_{i} \mid \widehat{\mathbf{H}}_{i}^{(k)}, \mathbf{x}_{j}^{i}\right)$ given as

$$
p\left(\mathbf{y}_{i} \mid \widehat{\mathbf{H}}_{i}^{(k)}, \mathbf{x}_{j}^{i}\right)=\frac{1}{\pi^{N}} \exp \left(-\left\|\mathbf{y}_{i}-\frac{\rho}{M} \widehat{\mathbf{H}}_{i}^{(k)} \cdot \mathbf{x}_{j}^{i}\right\|^{2}\right) .
$$

All the derivations provided in this article are brief due to length restrictions. Please refer to [6] for more details.

- M-step:

$$
\widehat{\mathbf{H}}_{i}^{(k+1)}=\arg \min _{\mathbf{H}_{i}} Q\left(\mathbf{H}_{i} \mid \widehat{\mathbf{H}}_{i}^{(k)}\right) .
$$

After some manipulations, the updated channel estimation $\widehat{\mathbf{H}}_{i}^{(k+1)}$ is obtained as

$$
\widehat{\mathbf{H}}_{i}^{(k+1)}=\mathbf{R}^{-1} \cdot \mathbf{U}^{H} \cdot \mathbf{Y}_{i}
$$

\section{- Initialization:}

We use the conventional minimal mean square error channel estimator for initialization, which is given by

$$
\widehat{\mathbf{H}}_{i}^{(0)}=\sqrt{\frac{\rho}{M}} \cdot \mathbf{S}_{\tau}^{H} \cdot\left(\frac{\rho}{M} \mathbf{S}_{\tau} \mathbf{S}_{\tau}^{H}\right)^{-1} \cdot \mathbf{Y}_{i}
$$

Soft extrinsic information of each coded bits is provided by taking $\widehat{\mathbf{H}}_{i}$ as the true channel coefficients followed by coherent MIMO detection,

$$
\begin{aligned}
L_{e x t}\left(c_{j, v}^{i}\right) & =\log \left(\frac{\Sigma_{\mathbf{x}_{j}^{i} \in \mathcal{D}_{j, v}^{+}} p\left(\mathbf{y}_{i} \mid \widehat{\mathbf{H}}_{i}, \mathbf{x}_{j}^{i}\right) \cdot p\left(\mathbf{x}_{j}^{i}\right)}{\sum_{\mathbf{x}_{j}^{i} \in \mathcal{D}_{j, v}^{-}} p\left(\mathbf{y}_{i} \mid \widehat{\mathbf{H}}_{i}, \mathbf{x}_{j}^{i}\right) \cdot p\left(\mathbf{x}_{j}^{i}\right)}\right) \\
& -\log \left(\frac{p\left(c_{j, v}^{i}=1\right)}{p\left(c_{j, v}^{i}=0\right)}\right), \quad 1 \leq i \leq L, \quad 12
\end{aligned}
$$

where

$$
0 \leq j \leq T_{d}, \quad 1 \leq v \leq\left(M \cdot \log _{2}|\mathcal{X}|\right) .
$$

It is well known that positive feedback generated by the short girth in an LDPC code directly affects the iterative message passing algorithm. With similar reasonings, correlations between input and output extrinsic information of the MIMO detector will also cause server system performance degradation. Therefore, in order to eliminate the 
existing correlation in the conventional EM-based detector, where $\widehat{\mathbf{H}}_{i}$ is a function of each input $L_{a p p}\left(\mathbf{x}_{k}^{i}\right)$ and causes positive feedback through (12), we propose the following modification,

$$
\widehat{\mathbf{H}}_{i}(j)=\widehat{\mathbf{H}}_{i}\left(\mathbf{Y}_{i}, \mathbf{S}_{\tau},\left\{L_{a p p}\left(\mathbf{x}_{k}^{i}\right)\right\}_{k \in \mathcal{E}}\right), 1 \leq j \leq T_{d},
$$

where $\widehat{\mathbf{H}}_{i}(j)$ is the channel estimation used to perform coherent detection for the $j^{\text {th }}$ coded vector $\mathbf{c}_{j}^{i}$, and $\mathcal{E}$ is a subset of $\left\{1,2, \cdots, T_{d}\right\}$ that excludes $j$, i.e. $j \notin \mathcal{E}$. The resulting APP EM algorithm remains the same except by setting $\left\{L_{a p p}\left(\mathbf{x}_{k}^{i}\right)\right\}_{k \in \overline{\mathcal{E}}}$ equal to zeros. Instead of having only one EM estimation, total of $\left\lceil T_{d} /\left(T_{d}-|\mathcal{E}|\right)\right\rceil$ separate EM estimations are to be completed during one entire decoding iteration in the modified EM-based detector.

\section{RECEIVER DESIGN OF THE CODED MIMO SYSTEM}

\subsection{LDPC-coded MIMO Receiver Structure}

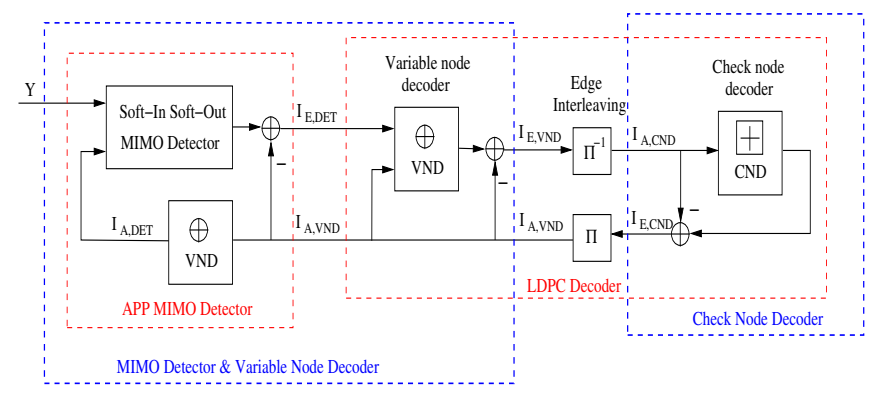

Fig. 2. Receiver structure of LDPC-coded MIMO systems

As demonstrated in Fig. 2, by connecting the soft MIMO detector and LDPC decoder together as one large iterative structure, conventional MIMO receiver actually consists of two iterative decoding loops. The soft extrinsic information, which describes the uncertainty of each coded bits, is iteratively exchanged in the outer loop between the MIMO detector and LDPC decoder as well as in the inner loop between variable node and check node decoders inside the LDPC decoder.

In this paper, we structure the MIMO receiver in another form by combining the soft MIMO detector and LDPC variable node decoder together as a super soft decoder, and hence closing the iterative decoding loop through exchanging extrinsic information between the super decoder and the LDPC check node decoder. The new receiver structure has a smaller decoding complexity compared with the conventional structure since it has only one iterative decoding loop. Furthermore, it has the advantage of converting the LDPC code optimization into a standard linear optimization approach, as shown in Section 4.3, greatly simplifying the optimal code searching algorithm.

\subsection{Extrinsic information transfer (EXIT) function}

In order to understand as well as design the iterative decoding systems having bipartite graph structures, we use the extrinsic information transfer characteristic of the soft MIMO detector and LDPC decoder proposed in [5], to analyze the convergence behavior of the iterative decoding schemes of the coded MIMO system.

By assuming the input a priori information $L_{a p p}(x)$ to the MIMO detector has a Gaussian distribution, the mutual information between the output extrinsic information $L_{e x t}(x)$ and the transmitted bits $x$, represented as

$$
I_{E, \text { det }}=I\left(L_{\text {ext }}(x) ; x\right)=F\left(\rho ; \sigma_{A}^{2}=J^{-1}\left(I_{A, \text { det }}\right)\right) \text {, }
$$

is obtained through Monte Carlo simulations (histogram measurements), where $J(\cdot)$ is the capacity of the binary input AWGN channel. Extrinsic mutual information $I_{E \text {,det }}$ is a function of both the system SNR $\rho$ and the noise variance level $\sigma_{A}^{2}$ of the input a priori information $L_{A p p}(x)$ (hence function of the input a priori mutual information $I_{A, d e t}$ ).

\subsection{LDPC Code Optimization}

Following the same reasonings given in [5] (for details see [6]), the extrinsic information transfer (EXIT) functions of the component MIMO soft decoders, including the super MIMO decoder (combination of MIMO detector and LDPC variable node decoder) and the LDPC check node decoder, are given by (refer to Fig. 2 for notations),

$$
\begin{aligned}
I_{E, v n d}\left(I_{A, v n d}\right) & =\sum_{i=1}^{D_{v}} \lambda_{i} \cdot J\left(\left(d_{v, i}-1\right) \cdot J^{-1}\left(I_{A, v n d}\right)\right. \\
& \left.+J^{-1}\left(\left.F\right|_{\rho}\left(I_{A, v n d}\right)\right)\right) \\
I_{E, \text { cnd }}\left(I_{A, c n d}\right) & \approx 1-\sum_{i=1}^{D_{c}} \rho_{i} \cdot J\left(\left(d_{c, i}-1\right) \cdot J^{-1}\left(1-I_{A, c n d}\right)\right)
\end{aligned}
$$

where $\lambda_{i}$ is the fraction of the variable nodes having edge degree $d_{v, i}, D_{v}$ is the number of different variable node degrees, and $\rho_{i}, d_{c, i}, D_{c}$ are similarly defined.

Successful decoding is assumed as long as the EXIT chart curve (15) is above curve (16) (with the $\mathrm{x}$ and $\mathrm{y}$ axises filpped). Therefore, instead of performing manual curve fitting to search for the optimal LDPC code [5], the LDPC code degree profile optimization can be reduced to the following maximization problem by taking the LDPC code rate $R$ as the objective

$$
\max _{\left\{\lambda_{i}, \rho_{i}\right\}} R=\max _{\left\{\lambda_{i}, \rho_{i}\right\}}\left(1-\frac{\sum_{i=1}^{D_{c}} \rho_{i} / d_{c, i}}{\sum_{i=1}^{D_{v}} \lambda_{i} / d_{v, i}}\right),
$$

under linear constraints given by

$$
\begin{aligned}
& I_{E, \text { vnd }}\left(I_{A, v n d}\right) \geq I_{A, \text { cnd }}\left(I_{E, \text { cnd }}\right)=I_{A, \text { cnd }}\left(I_{A, v n d}\right), \\
& \sum_{i=1}^{D_{v}} \lambda_{i}=1, \quad \sum_{i=1}^{D_{c}} \rho_{i}=1, \quad 0 \leq \lambda_{i}, \rho_{i} \leq 1 .
\end{aligned}
$$


The above optimization problem can be solved by an iterative search algorithm composed of two simple linear optimization steps [6].

\section{NUMERICAL AND SIMULATION RESULTS}

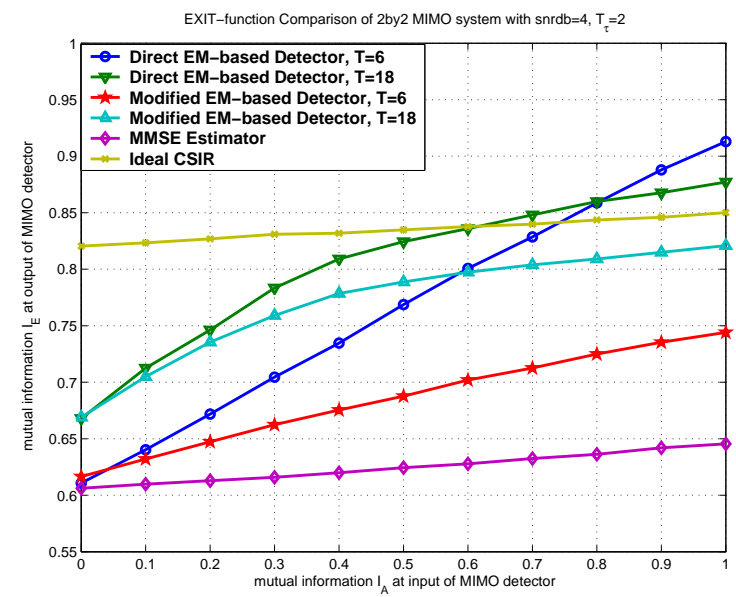

Fig. 3. Comparison of the extrinsic information transfer characteristic of different MIMO detectors.

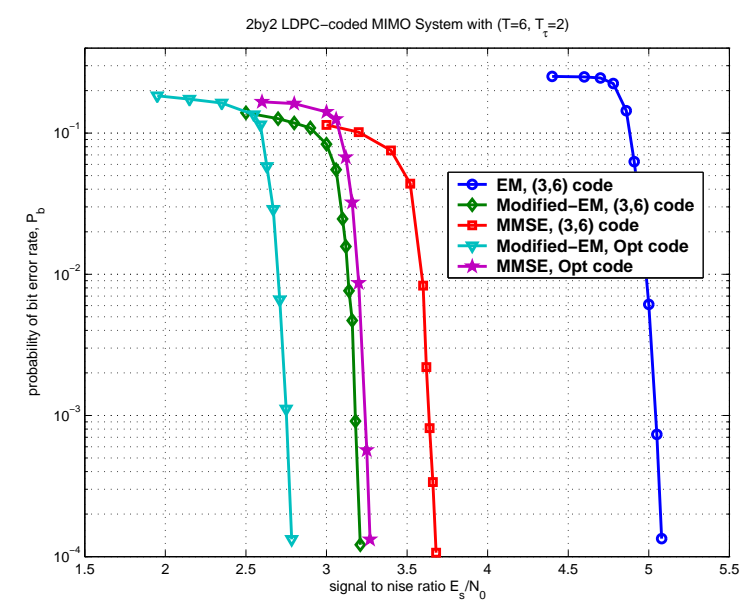

Fig. 4. Probability of bit error rate of a $2 \times 2$ rate $0.5 \mathrm{LDPC}$ coded MIMO system.

As an example, we demonstrate in Fig. 3 the extrinsic information transfer functions of the EM-based and modified EM-based soft MIMO detectors over an unknown $2 \times 2$ MIMO channel with coherent time interval $T=6$ and 18 , training length $T_{\tau}=2$, and signal to noise ratio $\rho=4 \mathrm{~dB}$. For comparison purpose, the mutual information transfer characteristics of the simple MMSE-based MIMO detector and detector with ideal CSI are also included in the plot. We can observe from the plot that for direct EM-based MIMO detector, output extrinsic mutual information $I_{E \text {, det }}$ is even greater than that of the detector with ideal CSIR in high
$I_{A, \text { det }}$ ranges, which directly indicates the existing positive feedback between the input and output extrinsic information. Such strong correlations between the output extrinsic information $L_{e x t}(x)$ and the input a priori information $L_{a p p}(x)$ will cause a severe performance degradation as shown in Fig. 4. Besides, it also can be observed that significant performance gain can be achieved by the modified EM-based MIMO detector compared with the MMSEbased detector especially when $T$ is large.

As verifications to the EXIT-Chart analysis, the actual system simulation results (average bit error rate) of a $2 \times 2$ MIMO system over unknown fading channel with coherent time $T=6$, and training number $T_{\tau}=2$ is demonstrated in Fig. 4. The outer LDPC code has a code rate $R=1 / 2$ and codeword length $n=8 \times 10^{4}$. As can be observed from the above plot, about $0.5 d B$ performance gain can be achieved by using the modified EM-based MIMO detectors than the MMSE-based detector, about $2 d B$ gain compared with the direct EM-based detector, and another $0.4 d B$ gain for code optimizations.

\section{CONCLUSION}

In this paper, we first propose a modified EM-based soft MIMO detector that can remove positive feedback between input and output extrinsic information completely. Based on the proposed detector, a novel iterative receiver structure is constructed with lower decoding complexity. Through the analysis of the extrinsic information transfer characteristic of the component soft decoders, a simple and efficient LDPC code optimization approach is also provided in this article. Numerical and simulation results are provided to further confirm the optimality of the proposed design approach for the coded MIMO system.

\section{REFERENCES}

[1] E. Telatar, "Capacity of multi-antenna gaussian channels," Tech. Rep., AT\&T Bell Labs, June 1995.

[2] F. Boixadera, J. J. Boutros and C. Lamy, "Bit-interleaved coded modulations for multiple-input multiple-output channels," IEEE Int. Symp. on Spread Spectrum Techniques \& App., NJ, Sept. 2000.

[3] J. Miguez, M. Gonzalez-Lopez, and L. Castedo, "Turbo aided maximum likelihood channel estimation for space-time coded systems," IEEE Int. Symp. on PIMRC 2002, Sept. 2002.

[4] F. Simoens, H. Wymeersch, and M. Moeneclaey, "Code-aided joint channel estimation and frame synchronization for mimo systems," in Workshop on SPAWC 2004, July 2004.

[5] S. ten Brink, G. Kramer, and A. Ashikhmin, "Design of lowdensity parity-check codes for modulation and detection," IEEE Trans. on Commun., vol. 52, pp. 670-678, Apr. 2004.

[6] J. Zheng and B. D.Rao, "On coding of MIMO systems with unknown channel state information," in preparation, 2004. 